\title{
COMMUNICATIONS
}

\section{Microwave lowpass filter with improved out-of-band performance based on tilted rectangular slots}

\author{
Shan-Shan Gao ${ }^{1,2,3}$, Jia-Lin $\mathbf{L i}^{4}$, Rong-Bin Chen ${ }^{5}$, Kexin Song ${ }^{6}$, \\ Yu-Shuang Liu ${ }^{1}$, Jia-Li Xu${ }^{1}$, Yue-Peng $\mathbf{L i}^{1}$
}

\begin{abstract}
A compact microwave lowpass filter with sharp selectivity and wide stopband based on tilted rectangular slots is proposed in this paper. The developed prototype filter is based on the log periodic theory, where tilted log-periodic rectangular slots are studied for the first time. Effects resulting from the tilted slots on the performance of the studied filter are discussed and conclusions are drawn. A prototype of the proposed lowpass filter was fabricated and measured. The measured results show that the lowpass filter features a high selectivity and a wide suppression band from $4.05 \mathrm{GHz}$ to $24.91 \mathrm{GHz}$ under a level of $19.2 \mathrm{~dB}$. Both measured and simulated performances are presented with good consistency.
\end{abstract}

K e y w o r d s: microwave filter, lowpass filter, rectangular slot, defected ground structure.

\section{Introduction}

With the rapid development of modern wireless communication systems, there is an increasing demand for microwave components with compact size and high performance [13]. In order to meet these requirements, the lowpass filter has been attracting increasing attentions due to its wide applications in RF systems. The traditional design of a microwave lowpass filter is based on transmission line like the microstrip to approximate the lowpass protype [4]. But this is difficult to achieve compact size, high selectivity, and wide stopband. To address this issue, different kinds of microwave lowpass filters with compact size, sharp cutoff and wide stopband had been investigated over the past years [5-11]. Among them, defected ground structure (DGS) had been extensively employed in the lowpass filter design to realize wide stopbands. In [9], a patterned ground was introduced to realize sharp roll-off. A. Boutejdar proposed a lowpass filter based on quasi-Yagi DGS [10] that exhibited a compact size, a sharp cutoff frequency, and a wide stopband bandwidth. In order to further improve the roll-off performance, interdigital shaped DGS was presented to achieve several transmission zeros near the cutoff frequency [11]. A modified complementary split-ring resonator was introduced to design the microwave lowpass filter with a compact size and a high selectivity [12]. By using a dumbbell-shaped DGS, a microwave lowpass filter with a sharp skirt and wideband suppressions was reported in [13]. In [14], a lowpass filter with a sharp transition from the passband to stopband was investigated.
In this paper, we investigated a set of tilted rectangular slots with log-periodic fashion for microwave lowpass applications. It is noted that the tilted slots with logperiodic fashion are, for the first time, studied here and some conclusions based on the tilted slots are drawn. By simulating analyzing the tilting angle of the rectangular slots, a microstrip lowpass filter with tilting angle of 30 degrees for the tilted slots and $3-\mathrm{dB}$ cutoff frequency at $3.24 \mathrm{GHz}$ is optimally designed, fabricated and tested. The studied filter characterizes a wide stopband under a 19.2-dB attenuation from $4.05 \mathrm{GHz}$ to $24.91 \mathrm{GHz}$. In addition, several transmission zeros near the cutoff frequency are realized to enhance the high selectivity. The measured performances for the fabricated filter show good consistency with the simulated results.

\section{Design of the tilted rectangular slots based lowpass filter}

The geometry of the proposed lowpass filter is shown in Fig. 1(a), where on the top, a $50 \Omega$ microstrip transmission line with a $0.76-\mathrm{mm}$ line-width is placed, while the back side is the metal ground but etched a set of tilted rectangular slots as shown in Fig. 1(b), between them, the layer is a substrate with a dielectric constant of 9.6 and a thickness of $0.8 \mathrm{~mm}$. Notice that the set of tilted rectangular slots are below the microstrip transmission line. Etching the set of grounded slots can disturb the shield current distribution on the ground plane, thus varying

\footnotetext{
${ }^{1}$ School of Electronic Information and Electrical Engineering, Chengdu University, Chengdu 610106, China, ${ }^{2}$ State Key Laboratory of Millimeter Waves, Nanjing 210096, China, ${ }^{3}$ Key Laboratory of Pattern Recognition and Intelligent Information Processing, Institutions of Higher Education of Sichuan Province, Chengdu University, Chengdu 610106, China, ${ }^{4}$ School of Physics, University of Electronic Science and Technology of China, Chengdu 610054, China, ${ }^{5}$ Department of Electronic and Information Technology, Jiangmen Polytechnic, Jiangmen 529030, China, ${ }^{6}$ Shanghai Aerospace Electronic Technology Institute, shanghai 201109, China, jialinli@uestc.edu.cn
}

https://doi.org/10.2478/jee-2021-0030, Print (till 2015) ISSN 1335-3632, On-line ISSN 1339-309X

(C) This is an open access article licensed under the Creative Commons Attribution-NonCommercial-NoDerivs License (http: //creativecommons.org/licenses/by-nc-nd/3.0/). 

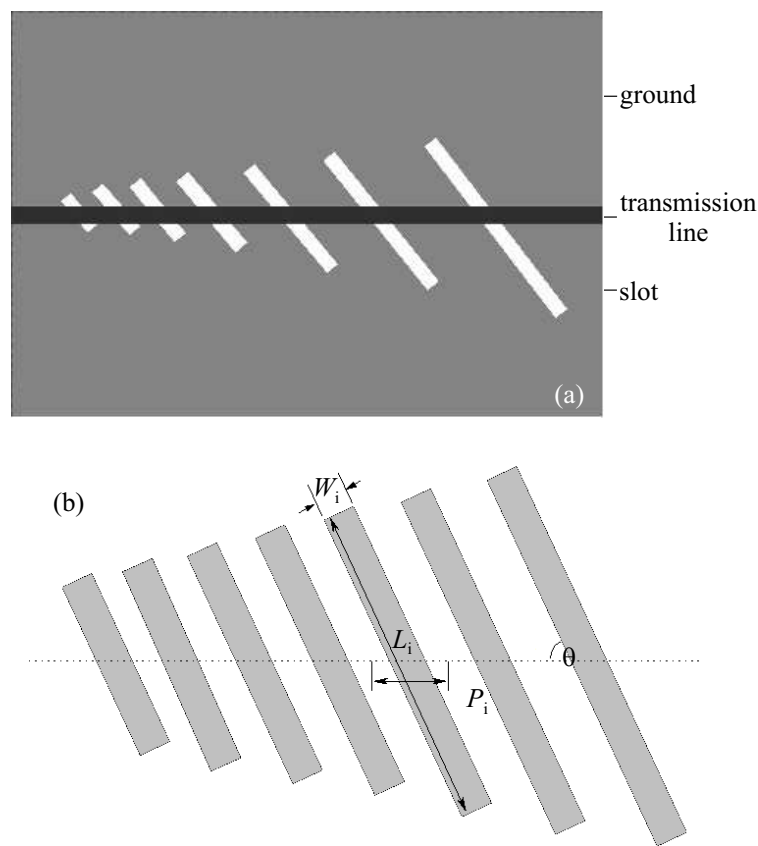

Fig. 1. Proposed lowpass filter: (a) - layout of the proposed lowpass filter, and (b) - tilted logperiodic rectangular slots

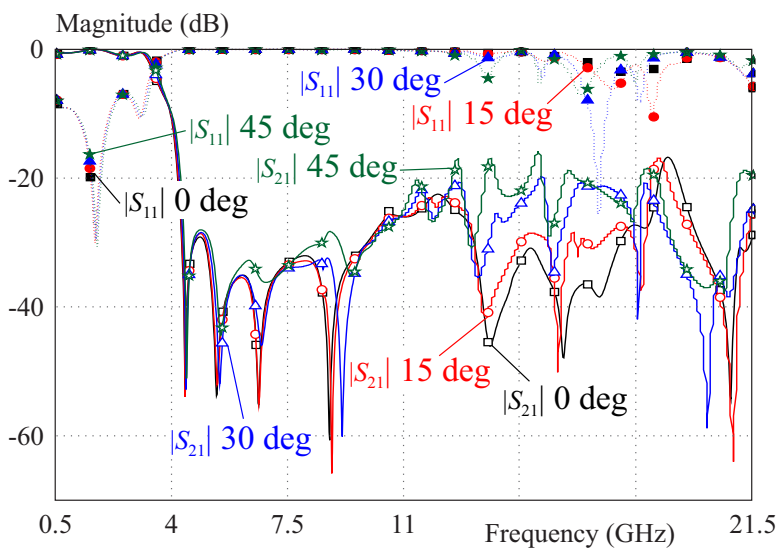

Fig. 2. Frequency responses of the studied lowpass filter under different tilted angles for $b=0$

the equivalent capacitance and inductance of the transmission line. The resonant frequency of such a structure depends on the physical dimensions, namely the length of each slot. Here the set of tilted rectangular slots are further placed based on the log periodic theory that is

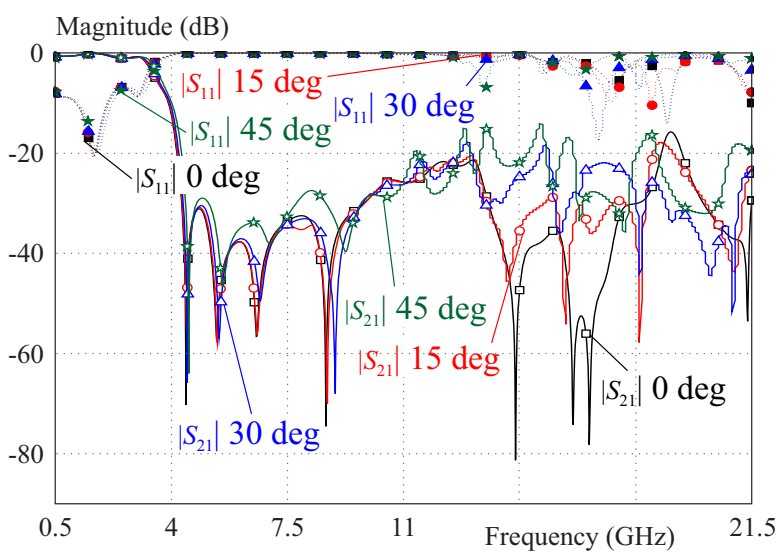

Fig. 3. Frequency responses under different tilted angles for $b=0.1$ first proposed for application to Yagi-Uda antenna [15] and recently studied for microwave filter application with enhanced performance [8]. Thus these rectangular slots are scaled in length and separation as well as slot-width to represent a $\log$ periodic pattern. Now for the $i^{\text {th }}$ slot shown in Fig. 1(b), $L_{i}$ represents its length, whereas $P_{i}$ indicates the distance between two adjacent slots, while $W_{i}$ denotes the slot width. Based on the log periodic theory, we have

$$
\frac{L_{i+1}}{L_{i}}=e^{a}, \quad \frac{W_{i+1}}{W_{i}}=e^{b}, \quad \frac{P_{i+}}{P_{i}}=e^{c},
$$

where $i=1,2,3 \ldots$, and $a, b, c$ can be positive or negative real but generally small in practice.

Now to in-depth study on the performance of such a tilted slot structure, parameter sweeping is carried out. Specifically, a tilted seven-slot array is discussed here and further, with some initial studies, we set the fourth slot as its length $L_{4}=10 \mathrm{~mm}$ and a width of $W_{4}=1.5$ $\mathrm{mm}$ together with its periodicity of $P_{4}=3.5 \mathrm{~mm}$ on the microwave substrate mentioned above. In the sweeping study, we are primarily interesting on the slot width $W_{i}$ and tilted slot angle $\theta$ on the electric performance under constant length and spacing periodicity. Thus $a=c=0.2$ are further set to be constants based on (1) and (3), and several index "b" that represents different slot width based on (2) is full-wave simulation studied under some possibly different tilted angles $\theta$.

Figure 2 shows that for constant slot-width, namely $b=0$, each slot has a width of $W_{i}=1.5 \mathrm{~mm}(i=$ $1,2, \ldots, 7)$ by tilting each slot with an angle of $\theta$ from 0 to 45 degrees, electric performance has different response and the primary difference lies in the out-of-band suppressions. It is seen from Fig. 2 that for the tilted angles $\theta=0$ and 15 degrees, the stopband suppressions for both cases exhibit peaks approximately $18.5 \mathrm{GHz}$. When further increasing the angle to 30 degrees, a wide suppression band referring to a suppression level of $20 \mathrm{~dB}$ is observed. If one increases the angle to 45 degrees, it is found several response peaks are generated in the band of interest under a $20 \mathrm{~dB}$ reference. On the other hand, the in-band responses are similar for all cases. The results indicate an optimal angle of $\theta=30$ degrees can effectively

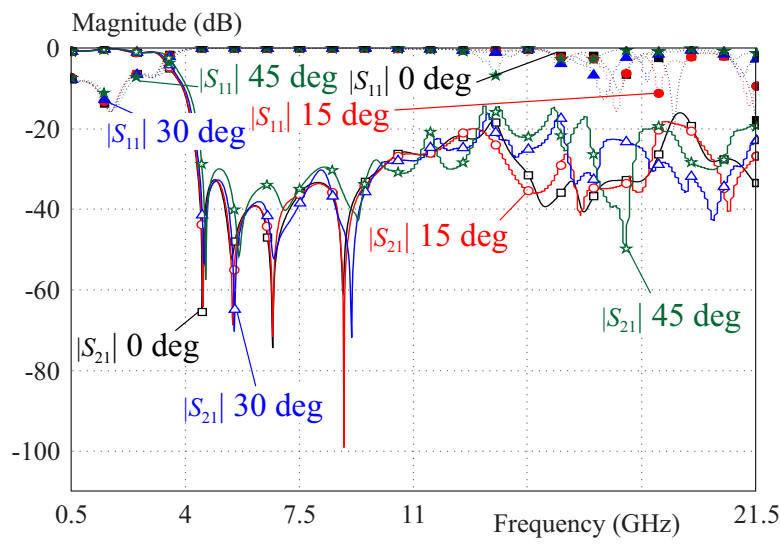

Fig. 4. Frequency responses under different tilted angles for $b=0.2$ 


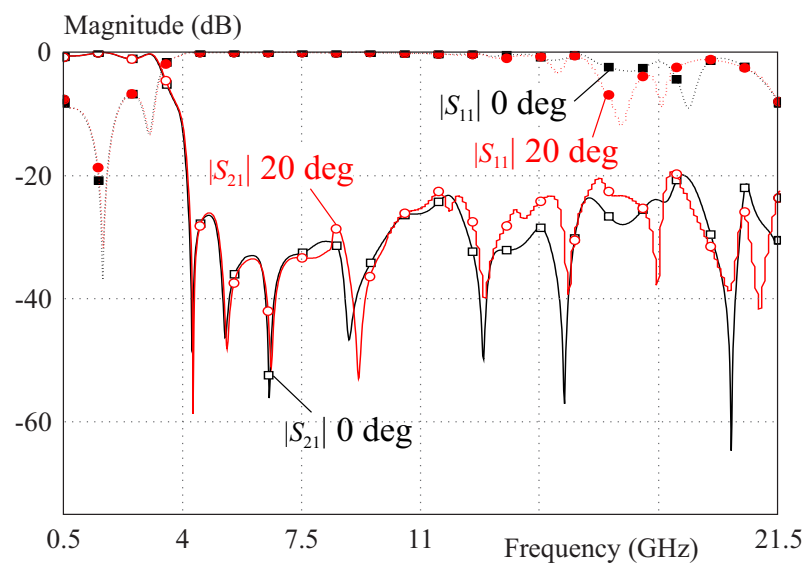

Fig. 5. Frequency responses for the tilted angles theta and orespective ly under constant slot width with $b=-1$

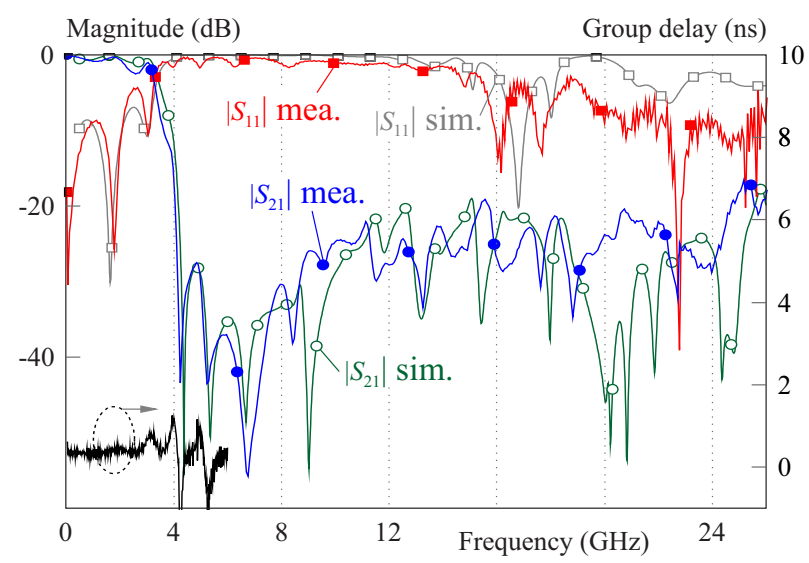

Fig. 6. Simulated and measured frequency responses of the proposed lowpass filter

disturb the surface current on the DGS ground within a wide frequency band. When deviating from the optimal tilted angle, current on the ground may exhibit some possible through path at some frequencies, resulting in the degraded suppression levels in the stopband. Therefore, as compared to the standard log-periodic DGS in [8], tilting the rectangular slots could further enhance the filter performance in terms of the suppression level in the stopband.
Based on above studies, varied slot widths with log periodic distributions are further numerically studied. Figs. 3 and 4 present the slot widths are positively increased from the left to right shown in Fig. 1 under the log periodic fashion. The results illustrate that for any tilted angles, either the small or the large angles (that is to say, $\theta=45$ degrees), the DGS ground can all create the possible current path to some extent in the studied stopband, leading to some response peaks as observed from Fig 3 and Fig. 4; this is also true for the case even without adopting the tilted slots (ie, $\theta=0)$.

Next, the slot width with negative distribution from the left to the right referred to Fig. 1 is studied. The numerically evaluated results are recorded in Fig. 5, where only the tilting angles for $\theta=0$ and 20 degrees are presented due to the fact that further increasing the tilting angle may make the slots on the left side to be overlapped. One can see for the negative index $b$ given by (2), general good suppression band can be realized as described in Fig. 5.

From these results, it could be seen the uniform slot width, namely $b=0$ in (2), could lead to wide suppression band with high suppression levels. In the following demonstrations, $b=0$ is used to design a prototype DGS filter.

\section{Developing a prototype DGS filter and results}

For demonstration purposes, a prototype microstrip lowpass filter based on above study is designed and developed. A total seven-slot with uniform slot width $(b=0)$ for each slot is utilized, and the fourth slot has a length of $L_{4}=10 \mathrm{~mm}$ with its periodicity $P_{4}=3.5 \mathrm{~mm}$. Both length ratio and periodicity ratio are of the same index of $\mathrm{a}=\mathrm{c}=0.2$, corresponding to each slot has a length of (units: mm) 5.4881, 6.7032, 8.1873, 10, 12.214, 14.9182 and 18.2212 from the left to the right in Fig. 1, respectively, while for each slot, it has a periodicity of 1.9209, 2.3462, 2.8656, 3.5, 4.2749, 5.2214 and 6.3774, respectively. The overall size is therefore $29.5835 \mathrm{~mm}$ $\times 16.53 \mathrm{~mm}$. Figure 6 shows the simulated and measured frequency responses of the proposed lowpass filter. The measured $3-\mathrm{dB}$ cutoff frequency is $3.24 \mathrm{GHz}$. This filter

Table 1. Comparisons among published and our proposed lowpass filter

\begin{tabular}{lccccc}
\hline \multirow{2}{*}{ Methods } & $\begin{array}{c}f_{\mathrm{c}-3 \mathrm{~dB}} \\
(\mathrm{GHz})\end{array}$ & $\begin{array}{c}\text { First attenuation } \\
\text { pole and level } \\
(\mathrm{dB}) /(\mathrm{GHz})\end{array}$ & $\begin{array}{c}\text { Stopband range and } \\
\text { suppression level } \\
(\mathrm{dB}) /(\mathrm{GHz})\end{array}$ & \multicolumn{2}{c}{$\begin{array}{c}\text { Substrate } \\
\varepsilon_{\mathrm{r}}\end{array}$} \\
\hline Slot DGS (8) & 3.07 & $52 / 4.16$ & $20 / 3.68-20$ & 9.6 & 0.8 \\
E shaped DGS (9) & 2.7 & $62 / 3.66$ & $35 / 3.39-7.37$ & 9.6 & 0.8 \\
Quasi-Yagi DGS (10) & 1.8 & $35 / 2.9$ & $20 / 2.8-10$ & 10.2 & 0.635 \\
Interdigital shaped DGS (11) & 3.11 & $22.5 / 3.23$ & $25 / 3.24-10.7$ & 10.2 & 1.27 \\
Complementary split-ring (12) & 3.37 & $46 / 4.3$ & $20 / 3.46-9.2$ & 3.66 & 0.508 \\
Dumbbell-shaped DGS (13) & 3.88 & $44.35 / 4.14$ & $20 / 4.07-15$ & 2.55 & 1.5 \\
Fan-shaped DGS (14) & 1.49 & $57.2 / 2$ & $20 / 1.65-7.41$ & 10.2 & 0.635 \\
In this work & 3.24 & $43.4 / 4.24$ & $19.2 / 4.05-24.91$ & 9.6 & 0.8 \\
\hline
\end{tabular}


has a stopband from 4.05 to $24.91 \mathrm{GHz}$ with suppression level greater than $19.2 \mathrm{~dB}$ from measurements. In addition, several transmission zeros near the cut-off frequency appear at $4.24,5.23$, and $6.76 \mathrm{GHz}$, thus improving the selectivity of the filter. Meanwhile, the measured group delay is approximately $0.4 \mathrm{~ns}$ in the passband. Eventually, for performance comparisons, Table 1 lists some reported designs and this proposed filter. It is seen the proposed lowpass filter is simple both in structure and in design, and it also characterizes good out-of-band performances.

\section{Conclusions}

In this paper, a set of tilted rectangular slots with logperiodic fashion has been studied for the first time and proposed for the design of microstrip lowpass filter. The proposed tilted rectangular slots can not only generate attenuation pole near the cutoff frequency, but also exhibit a wide stopband bandwidth with high suppression levels. A demonstrator filter has been optimally designed, developed and examined with good consistency between simulations and measurements. Its enhanced selectivity and out-of-band performance are attractive in modern wireless communications.

\section{REFERENCES}

[1] S.-S. Gao, S. Sun, J.-L. Li, and T. Yan, "Compact dual-mode dual-band bandpass filter with inside-outside-reversed dual-ring topology,", Electron. Lett,.

[2] L. Xia, J.-L. Li, B. A. Twumasi, P. Liu, and S.-S. Gao, "Planar dual-band branch-line coupler with large frequency ratio,", IEEE ACCESS,

[3] M. Yao, J. L. Li, L. Xia, and S. S. Gao, "Wide dual-band dual-circularly polarized holographic metasurface,", Journal of Physics D: Applied Physics,

[4] J. S. Hong, Microstrip filters for RF/microwave applications, 2nd, New York, Wiley, 2011.

[5] C.-J. Chen, C.-H. Sung, and Y.-D. Su, "A multi-stub lowpass filter,", IEEE Microw. Wireless Compon. Lett,.

[6] S. M. H. Mousavi, S. V. A.-D. Makki, H. Siahkamari, S. Alirezaee, and M. Ahmadi, "Performance improvement of microstrip LPF based on transfer function analysis,", IEEE Microw. Wireless Compon. Lett,

[7] G. Moloudian, S. Bahrami, and R. M. Hashmi, "A microstrip lowpass filter with wide tuning range and sharp roll-off response,", IEEE Trans Circuits Syst. II, Exp. Briefs,.

[8] J. Gong, "A rectangular slot Yagi DGS microstrip filter with sharp rolloff and wide suppression band,", Journal of Electrical Engineering,.

[9] W. Shao and J.-L. Li, "Accurate modeling of a patterned ground and its application to microwave filters", Applied Computational Electromagnetics Society Journal,.

[10] A. Boutejdar, "Design of broad-stop band lowpass filter using a novel quasi-Yagi-DGS-resonators and metal box-technique,", Microw. Opt. Technol. Lett,.

[11] S. Liu, J. Xu, and Z. Xu, "Sharp roll-off lowpass filter using interdigital DGS slot", Electron. Lett, vol. 51, no. 17, pp. 1343-1345, 2015.
12] M. Xiao, G. Sun, and X. Li, "A lowpass filter with compact size and sharp roll-off,", IEEE Microw. Wireless Compon. Lett,.

13] P. Zhang and M. Li, "A novel sharp roll-off microstrip lowpass filter with improved stopband and compact size using dual-plane structure,", Microw. Opt. Technol. Lett,.

14] A.-O. Ertay, M. Abbak, and S. Simsek, "An improved stopband and sharp roll off microstrip low pass filter with defected ground structures,", International Journal of Microwave and Wireless Technology,.

[15] N. Barbano, "Log periodic Yagi-Uda array,", IEEE Trans. Antennas. Propag,

Received 19 January 2021

Shan Shan Gao was born in Sichuan Province, China. She received the $\mathrm{PhD}$ degree in radio physics from the University of Electronic Science and Technology of China (UESTC), Chengdu, in 2012. From June 2011 to July 2013, she was a Research Assistant at the Department of Electrical \& Electronic Engineering, the University of Hong Kong. She has been the Associate Professor at the School of Electronic Information and Electrical Engineering, Chengdu University.

Jia-Lin Li was born in Sichuan, China. He received the MS degree from the University of Electronic Science and Technology of China (UESTC), Chengdu, China, in 2004, and the $\mathrm{PhD}$ degree from the City University of Hong Kong, Hong Kong, in 2009, both in electronic engineering. From September 2005 to August 2006, he was a Research Associate with the Wireless Communication Research Center, City University of Hong Kong, Hong Kong. Since September 2009, he has been with the School of Physical Electronics, UESTC, where he is currently a Professor. His research interests include microwave/ millimeter-wave antenna and arrays, circuits and systems, interactions between microwave and complex medium, and so on.

Rong-Bin Chen was born in January 1980 at Guangdong, China. He received BS Degree from the Shantou University, Shantou, China, in 2003, and MS Degree from the Sun Yat-sen University, Guangzhou, China, in 2005. From July 2005 to June 2018, he was with the Jiangmen Branch of China Mobile Group Guangdong Co., Ltd., Jiangmen, China, where he served as a Technique Supporter for maintenance, construction and development of $2 \mathrm{G} / 3 \mathrm{G} / 4 \mathrm{G} / 5 \mathrm{G}$ wireless/mobile networks. Since June 2018, he has been with the Department of Electronic and Information Technology, Jiangmen Polytechnic, Jiangmen, China. His research interests include wireless communication technology, internet of things, and so on.

Kexin Song was born in Tianjin, China. He received the $\mathrm{PhD}$ degree in Astrometry and Celestial Mechanics from Natinal Time Service Center, Chinese Academy of Sciences (NTSC, CAS), Xi'an, in 2011. From July 2011 to nowadays, he works as a chief designer and has been a senior engineer at the Shanghai Aerospace Electronic Technology Institute.

Yu Shuang Liu was born in Sichuan Province, China. $\mathrm{He}$ is an undergraduate student at the School of Electronic Information and Electrical Engineering, Chengdu University.

Jia Li Xu was born in Sichuan Province, China. She has been the Associate Professor at the School of Electronic Information and Electrical Engineering, Chengdu University.

Yue Peng Li has been the experimentalist at the School of Electronic Information and Electrical Engineering, Chengdu University. 